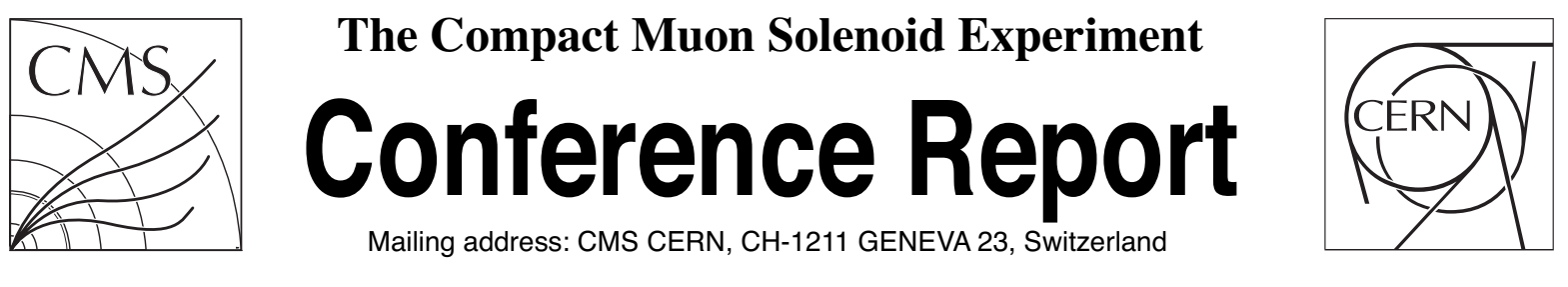

23 March 2010 (v4, 26 March 2010)

\title{
DC-DC Conversion Powering Schemes for the CMS Tracker at Super-LHC
}

Katja Klein, Lutz Feld, Rüdiger Jussen, Waclaw Karpinski, Jennifer Merz and Jan Sammet

\begin{abstract}
The CMS experiment at the Large Hadron Collider (LHC) at CERN, Geneva, houses the largest silicon strip tracker ever built. For the foreseen luminosity upgrade of the LHC, the Super-LHC, however, a completely new silicon tracker will have to be constructed. One out of several major improvements currently under consideration is the implementation of a track trigger, with tracking information being provided to the first level trigger. Such an intelligent tracker design, utilising fast digital readout electronics, will most certainly lead to an increased power consumption, compared to today's tracker. In combination with the desire to reduce the amount of passive material inside the tracking volume and the impracticality to exchange or even add additional supply cables, a novel powering scheme will be inevitable. In this article a powering scheme based on DC-DC conversion is proposed, and requirements for the DC-DC converters are discussed. Studies of important DC-DC converter quantities such as the power efficiency, conducted and radiated noise levels, and material budget are presented, and a possible implementation of DC-DC buck converters into one proposed track trigger layout is sketched.
\end{abstract}

Presented at WIT2010: WIT2010 Workshop on Intelligent Trackers 


\section{Contents}

1. Introduction 囵

2. DC-DC converters for track trigger layers

2.1 Radiation-hard technology and DC-DC converter development

2.2 Power requirements and efficiency 3

2.3 Conducted and radiated noise

2.4 Material budget, space requirements and integration aspects

3. Summary 5

\section{Introduction}

The Super-LHC (SLHC) is a potential luminosity upgrade of the LHC. The plan is to increase the luminosity in several steps, with the ultimate goal of $10^{35} \mathrm{~cm}^{-2} \mathrm{~s}^{-1}$, to be reached around 2020 .

Replacements for the CMS [U] silicon pixel detector and silicon micro-strip tracker are currently under design. The new detectors will have to cope with much higher radiation levels, and an increase in the number of pile-up events per bunch-crossing by a factor of about 20. The new strip tracker therefore has to be of higher granularity, to keep the occupancy under control. To maintain the first level trigger rate at its design value of $100 \mathrm{kHz}$, tracker information will have to be used in the Level-1 (L1) trigger. This requires a completely new tracker concept. Due to the larger channel count and the increased functionality it is quite likely that the front-end (FE) power consumption of the future strip tracker will exceed significantly today's value of $34 \mathrm{~kW}$. Smaller feature size semi-conductor processes will lead to power savings, but require lower supply voltages and thus larger currents, increasing the resistive losses in supply cables. More or thicker cables cannot be installed, as cable channels are hardly accessible and are also already full. The reduction of material, including power cables, inside the tracking volume itself is one of the goals of the tracker upgrade. A novel powering scheme is therefore needed, and after a review process a DCDC conversion powering scheme was chosen in 2009 as the baseline solution for the CMS tracker. The idea behind DC-DC conversion is simple: since $P=V \cdot I=(r \cdot V) \cdot(I / r)$, with the conversion ratio $r>1$, the power can be supplied at a higher voltage, but lower current. This reduces Ohmic losses in supply cables and the amount of copper needed inside the tracker volume. Step-down converters close to the FE electronics transform the input voltage $V_{\text {in }}=r \cdot V_{\text {out }}$ to $V_{\text {out }}$. These converters can be based on inductors or switched capacitors. For the application in track trigger layers the buck converter (Fig. [1, left) is well suited: with relatively few components it can operate at high $r$, provide currents of several Amperes, with an efficiency of up to $80 \%$. Two power transistors are alternately switched on and off, an inductor acts as energy storage element, and input and output filter networks shunt AC components. A feedback control loop regulates the duty 

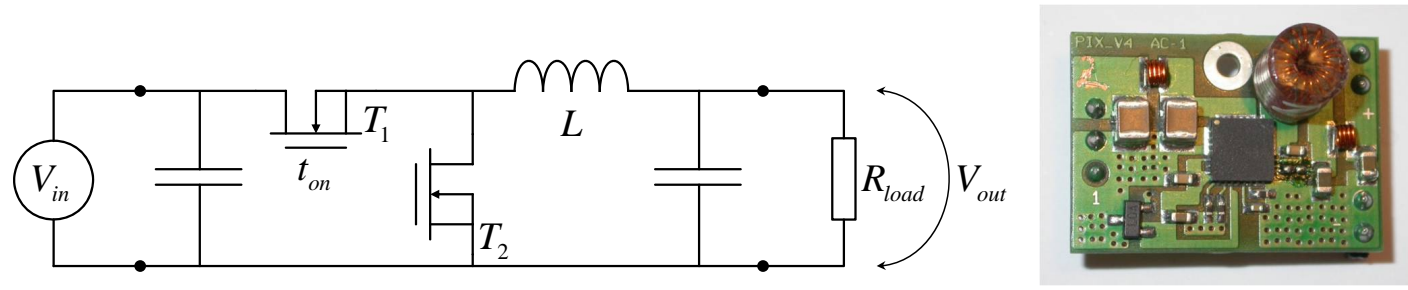

Figure 1. Left: simplified schematics of a buck converter. The feed-back regulation circuit based on Pulse Width Modulation is not shown. Right: photograph of the AC_AMIS2 buck converter.

cycle and thus the converter's output voltage.

There are many challenges to be mastered. The power transistors must stand the input voltage (up to $12 \mathrm{~V}$ ), and be sufficiently radiation-tolerant to survive SLHC conditions. High-frequency switching might inject conductive noise into the detector system. Air-core inductors, needed because of saturation of ferrite cores in the 3.8 T magnetic field of CMS, might radiate electro-magnetic noise. The converter's size and mass must be reduced as much as possible. A low efficiency would cancel out the advantages of the concept.

\section{DC-DC converters for track trigger layers}

Several suggestions for the implementation of a track trigger have been put forward. All are based on the discrimination between low and high transverse momentum $\left(p_{T}\right)$ tracks to reduce the amount of information that needs to be transmitted off-detector, by exploiting the bending of tracks in the magnetic field [2]. Ideas include the $p_{T}$ measurement via the cluster width or in closely $(1-2 \mathrm{~mm})$ spaced detector layers (stacked layers) [3]. Pixelated detectors are used in the latter approach. The resulting 2-hit track pieces are forwarded to the L1 trigger. Power-hungry, fast digital electronics would be needed to correlate hit positions within one stack. Layout suggestions range from one stacked layer combined with a classical strip tracker, to a tracker constructed exclusively from stacked layers, arranged as double-stacks [四].

Stacked layers present a particularly challenging environment for DC-DC converters, as will be detailed in the following.

\subsection{Radiation-hard technology and DC-DC converter development}

DC-DC converters have to cope with an expected dose of about $1.3 \mathrm{MGy}$ and a 1-MeV-neutronequivalent fluence of about $9 \cdot 10^{15} \mathrm{~cm}^{-2}$ (for a radius $\approx 20 \mathrm{~cm}, z \approx 3 \mathrm{~m}$, an integrated luminosity of $5000 \mathrm{fb}^{-1}$, with a safety factor of 2). A group at CERN has evaluated the radiation tolerance of several candidate technologies. Two of them seem to be sufficiently radiation-tolerant, with one performing a bit better than the other. Buck converter ASIC prototypes in both technologies have been developed by the same group [5]. Their AMIS2 ASIC, the second prototype in a $0.35 \mu \mathrm{m}$ high voltage technology, has been used to develop a low-mass, low-noise DC-DC converter (AC_AMIS2, Fig. 1, right). Its operating parameters are: $V_{\text {in }}<12 \mathrm{~V} ; V_{\text {out }}=1.2,2.5$ or $3.3 \mathrm{~V}$; $r \leq 10$; and $I_{\text {out }} \leq 3 \mathrm{~A}$. The switching frequency $f_{s}$ is either fixed to $1.3 \mathrm{MHz}$, or - for test purposes programmable with a potentiometer from $0.6-4.0 \mathrm{MHz}$. The coil is a custom-made air-core toroid 
with an inductance of $L \approx 600 \mathrm{nH}$, a diameter of $6 \mathrm{~mm}$ and a height of $7 \mathrm{~mm}$. The PCB has an area of $18 \times 25 \mathrm{~mm}^{2}$ and is equipped with Pi-filters (a type of noise filter) at the input and output.

For comparison, DC-DC converters with non-radiation-hard commercial chips of type EQ5382D by Enpirion have been developed (AC2) [ [6]. The operating parameters are different: $V_{\text {in }} \leq 5.5 \mathrm{~V}$ (7 V max.), $I_{\text {out }} \leq 0.8 \mathrm{~A}$, and $f_{s} \approx 4 \mathrm{MHz}$. This board is equipped with the same inductor, but filter capacitors only, i.e. no Pi-filters. The area is $12 \times 19 \mathrm{~mm}^{2}$.

\subsection{Power requirements and efficiency}

The operating parameters of future DC-DC converters for track trigger layers are not yet known in detail, but plausible ranges and upper limits can be given. The estimated FE power consumption per unit area is $100 \mathrm{~mW}$ per $\mathrm{cm}^{2}$; estimates for the total power consumption go up to $100 \mathrm{~kW}$. From a maximal current of about $15 \mathrm{kA}$ allowed inside the water-cooled cable channels it can be deduced that a conversion ratio of up to 10 might be required. The power per stacked module will be in the range of 2-9 W. Assuming a feature size of $130 \mathrm{~nm}$ or below, the analogue supply voltage is $1.2 \mathrm{~V}$; the digital can be lower. Operating the digital part of the circuit at $0.9 \mathrm{~V}$ would reduce the digital power consumption (at least $50 \%$ of the total) to half its value at $1.2 \mathrm{~V}$. The current requirements for a stacked module amount then up to $4 \mathrm{~A}$ each at $1.2 \mathrm{~V}$ and $0.9 \mathrm{~V}$. A likely scenario is the deployment of two buck converters per stacked module (digital currents are too large to use capacitor-based converters, and linear regulators introduce an efficiency penalty of $25 \%$ ).

A high efficiency, $P_{\text {out }} / P_{\text {in }}$, is crucial for the successful deployment of DC-DC converters. Figure $\square$ shows the measured efficiency of the AC_AMIS2 converter, as a function of input voltage and output current. For a conversion ratio of 3 and currents of about $1 \mathrm{~A}$, the efficiency is around $75 \%$. The efficiency decreases for higher conversion ratios and currents, dropping to 50-55\% in the parameter region required for stacked layers.

Inefficiencies from resistive losses, e.g. in transistors $\left(R_{o n}\right)$, wire bonds, and the coil $\left(R_{D C} \approx\right.$ $80 \mathrm{~m} \Omega$ ), rise with the current ripple $\Delta I \propto 1 / f_{s}$; switching and driving losses rise with $f_{s}$. The efficiency is thus a strong function of the switching frequency, and peaks for the AC_AMIS2 converter at $1.0 \mathrm{MHz}$. Significant improvements are expected e.g. from operating at the optimal frequency, from flip-chip interconnect technology, and from lower operating temperatures.
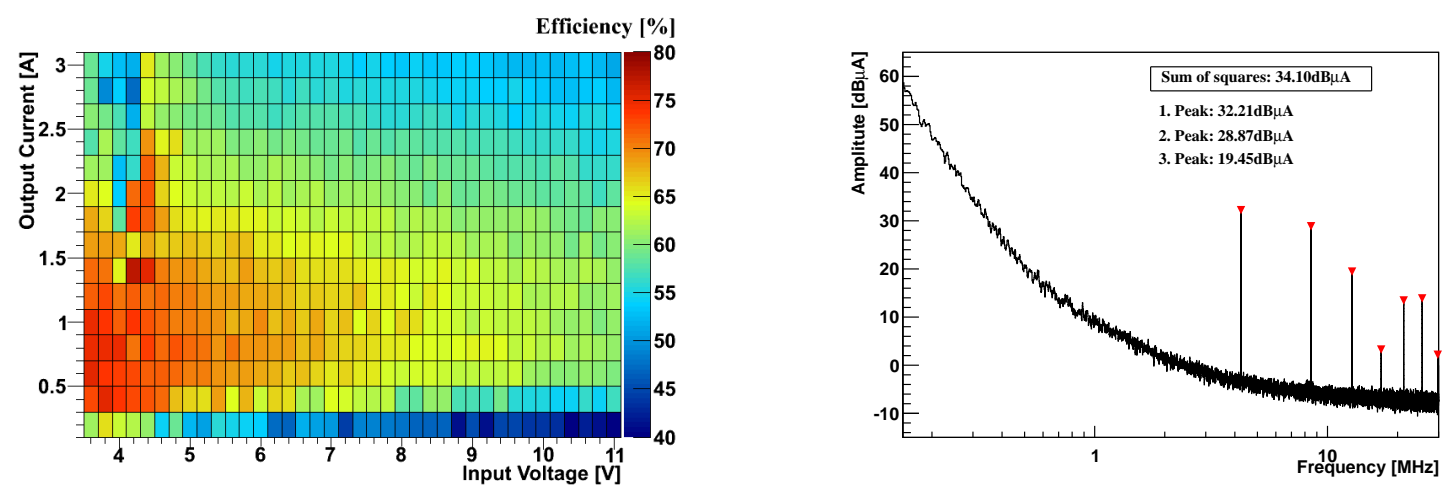

Figure 2. Left: efficiency of the AC_AMIS2 converter, for $V_{\text {out }}=1.2 \mathrm{~V}$ and $f_{s}=1.3 \mathrm{MHz}$, as a function of input voltage and output current. Right: DM noise spectrum of the AC2 converter, $V_{\text {in }}=5.5 \mathrm{~V}, V_{\text {out }}=1.3 \mathrm{~V}$. 


\subsection{Conducted and radiated noise}

DC-DC converters are switching large currents with $\mathrm{MHz}$-frequencies. This leads to a current ripple $\Delta I=\left(V_{\text {in }}-V_{\text {out }}\right) \cdot\left(V_{\text {out }} / V_{\text {in }}\right) \cdot\left(1 / f_{s}\right) \cdot(1 / L)$ in the inductor, and an output voltage ripple $\Delta V$ due to the finite output capacitance and its Equivalent Series Resistance ESR $\propto \Delta I /\left(f_{s} \cdot C\right)+E S R \cdot \Delta I$. The voltage ripple manifests itself as conducted noise, propagating through the cables, while the current ripple leads to noise emissions by the changing magnetic near field of the coil.

The conductive noise spectra in Common and Differential Mode (CM, DM) can be measured by picking up the noise current in the cables with a calibrated current probe connected to a spectrum analyzer. The DM spectrum of $\mathrm{AC} 2$, for example, (Fig. 2, right) shows a clear peak at the switching frequency, but the higher harmonics are also present, though with a lower amplitude.

To understand the relevance for a concrete detector system, its susceptibility has to be taken into account. System tests with a current CMS tracker end-cap substructure with four silicon strip modules have been performed. The APV25 readout ASIC is an analogue 128-channels chip with a shaping time of $50 \mathrm{~ns}$. Two converters are integrated close to the FE-hybrid and provide the supply voltages of 1.25 and $2.5 \mathrm{~V}$. The set-up and analysis method are described in detail in [6].

The conductive DM noise increases the module noise level, in particular on module edge channels, which are most sensitive, as explained in [6]. The quadratic sum of the noise of strips 1 and 512 is thus used as the most sensitive observable to characterize the noise behaviour. Magnetic emissions lead to a distortion of the shape of the noise distribution, with higher noise on APV-edge channels. The conductive noise has been systematically studied both for the AC2 and the AC_AMIS2 converters. As expected, increasing the conversion ratio increases the noise. In addition, with the AC_AMIS2 more noise is observed for lower switching frequencies (which provide a higher efficiency). Filtering is therefore required. The $\mathrm{AC} 2$ converter has been operated with external Pi-filters, with great success: no significant extra noise has been measured for the whole accessible input voltage range (Fig. [3, left). The AMIS_2 converter shows equally low noise as the AC2 when operated under the same conditions (Fig. 3, right). For the working conditions required for stacked layers, i.e. large $r$ and low $f_{s}$, the noise level is not yet satisfactory, and further work is needed. A scanning table equipped with a pick-up probe is used to measure the distribution of the magnetic field above the DC-DC converter. The magnetic field above the toroid has been found to be of
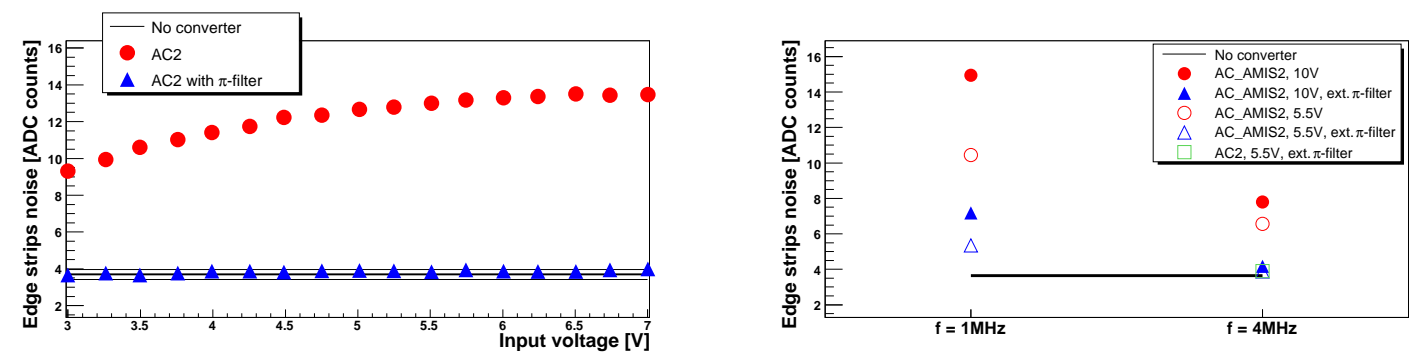

Figure 3. Left: edge strips noise without converter (black line with error band), and AC2 converter with (triangles) and without (circles) Pi-filter. Right: edge strips noise of the AC2 converter (box) and AMIS2 converters with $10 \mathrm{~V}$ (filled symbols) and $5.5 \mathrm{~V}$ input voltage (empty symbols); without (circles) and with (triangles) external Pi-filter. 
multi-polar shape, probably due to a superposition of the field of a perfect toroid with the field of a single wire loop, formed by the first and last half-turn. This shape was reproduced with Finite Element simulation. Work is ongoing to optimize the coils for reduced emission.

\subsection{Material budget, space requirements and integration aspects}

The effect of DC-DC conversion on the tracker end-cap (as an example) material budget was studied with a GEANT4-based simulation within the CMS software CMSSW, for the following scenario: one buck converter with the components and dimensions of the AC2 per silicon module, integrated onto the FE-hybrid, $80 \%$ efficiency, $r=8, I_{\text {out }}=2 \mathrm{~A}, V_{\text {out }}=1.2 \mathrm{~V}$. The buck converters add about $10 \%$ of the material of current strip modules, but the net effect, when the reduction of copper cross-sections in power cables and on motherboards is taken into account, is a saving of $31 \%$ in the category of electronics and cables, and $8 \%$ on the total end-cap material budget [6].

The space required by DC-DC converters can be estimated on the basis of the current boards: 19 $\mathrm{x} 12 \mathrm{~mm}^{2}$ for the AC2 board (no Pi-filters), and $25 \times 18 \mathrm{~mm}^{2}$ for the AMIS2 board (some components will later be integrated on-chip). It will be difficult to reduce the area below $2 \mathrm{~cm}^{2}$ without compromising efficiency and noise immunity. Two converters can be integrated onto one PCB, which will increase its area by a factor of 1.5 or less. The height is currently about $10 \mathrm{~mm}$.

Silicon modules proposed for stacked layers are typically small, e.g. $80 \mathrm{~mm}$ x $26 \mathrm{~mm}$ [3]. In particular in designs with data transmission between layers in the bulk of the module, readout ASICs cover most of the sensor area, leaving no space for passives. A narrow strip at the module edge is typically reserved for auxiliary chips. While space on the module is tight, integration onto the support structure is an alternative. In the double-stack proposal [ [W], stacked modules are installed on top and bottom of a hollow beam structure. Converters could be integrated onto the side faces of, or inside, this beam. This would also ease cooling and shielding of the converters.

\section{Summary}

A DC-DC conversion powering scheme is foreseen for the CMS tracker upgrade, with the most demanding requirements in terms of conversion ratio, output currents and space constraints coming from the stacked layer designs proposed for the implementation of a track trigger. First measurements of efficiency and noise with a radiation-tolerant prototype are encouraging, but more work is needed to make these devices compatible with the requirements of a future CMS tracker.

\section{References}

[1] The CMS Collaboration, The CMS experiment at the CERN LHC, 2008 JINST 3 S08004.

[2] K. Klein, The Upgrade of the CMS Tracker for Super-LHC, POS (RD 0 9) 034, and references therein.

[3] G. Hall, Design of a trigger module for the CMS Tracker at SLHC, CERN-2009-006 (2009).

[4] M. Mannelli, Intelligent trackers as L1 trigger providers for the SLHC, these proceedings.

[5] S. Michelis et al., ASIC buck converter prototypes for LHC upgrades, TWEPP-09, Paris, France (2009).

[6] K. Klein et al., Experimental studies towards a DC-DC conversion powering scheme for the CMS silicon strip tracker at SLHC, CERN-2009-006 (2009); and System tests with DC-DC converters for the CMS silicon strip tracker at SLHC, CERN-2008-008 (2008). 